\title{
Adjuvant therapy in colorectal cancer - studies of the FOGT group
}

\author{
Ludger Staib ${ }^{1 *}$, Marko Kornmann², Karl-Heinrich Link ${ }^{3}$ \\ From 16th International Charles Heidelberger Symposium on Cancer Research \\ Coimbra, Portugal. 26-28 September 2010
}

The FOGT (Study Group for GI-Oncology) was formed among clinicians and scientists with the aim of testing innovative scientific concepts in gastrointestinal tumors, especially colorectal cancers, within controlled clinical studies.

In the FOGT-1 trial $(\mathrm{n}=813$ stage III or II(T4N0) colon cancer patients) the effect on recurrence-free and overall survivalwas tested by an adjuvant chemotherapy protocol with double-modulation of 5-fluorouracil (5-FU) and levamisole (LEV) with either folinic acid (FA) or interferon-alpha (IFNa) in a three arm randomized trial within 60 German cancer centers. In parallel, in the FOGT-2 trial stage II or III rectal cancer patients ( $\mathrm{n}=$ 796 pts.) were treated with additional postoperative radiochemotherapy (50,4 Gy). The most effective arm of these trials was tested as control arm versus a 5-FU/FA/ irinotecan (FOLFIRI) protocol in the two-arm FOGT-4 trial ( $\mathrm{n}=281$ stage III or II(T4N0) colon cancer pts.) for adjuvant therapy in colon cancer.

In the FOGT-1 trial, we found a $11 \%$ overall survival (OAS) benefit in patients who were treated for one year adjuvantly with 5 -FU plus FA (77\% OAS), compared to treatment with either 5 -FU or 5-FU plus interferonalpha (each 66\% OAS). This effect was statistically significant for colon cancer patients (FOGT-1), but not for rectal cancer patients (FOGT-2). In rectal cancer, the 5 -FU plus FA arm showed this effect with a $12 \%$ survival benefit only in the stage II subgroup. The FA modulation of 5-FU was safe and cost-effective. The modulation with IFNa was less effective and caused more toxicity, mainly diarrhea in rectal cancer patients. When the 5FU/FA protocol was slightly modulated (treatment for 6 months, no LEV) and compared to the
FOLFIRI protocol (FOGT-4), survival and toxicity in the 5FU/FA arm was similar to the toxicity observed in the earlier studies, and the FOLFIRI arm was significantly more toxic ( $40 \%$ vs. $14 \%$ grade III/IV toxicity) without any benefit in recurrence rates ( $25 \%$ vs. $23 \%$ local/systemic recurrence) or survival. The FOGT-4 study confirms three other studies that - in contrast to palliative treatment protocols - did not find any benefit for the role of irinotecan in the adjuvant treatment of colon cancer.

In conclusion, by conducting the three FOGT-studies with more than 1800 patients, we identified a safe and effective treatment protocol for adjuvant therapy in colon cancer, consisting of infusional 5-fluorouracil and folinic acid, given for at least six months, and well combinable with additional postoperative radiotherapy. This protocol was not improved by addition of irinotecan in colon cancer patients.

\section{Author details}

${ }^{1}$ Department of General and Visceral Surgery, Klinikum Esslingen, Esslingen, Germany. ${ }^{2}$ Department of General, Visceral and Transplantation Surgery, University of Ulm, Ulm, Germany. ${ }^{3}$ Oncology and Special Visceral Surgery, Asklepios Paulinen Klinik, Wiesbaden, Germany.

Published: 24 September 2010

\section{doi:}

Cite this article as: Staib et al:: Adjuvant therapy in colorectal cancer studies of the FOGT group. BMC Proceedings 2010 4(Suppl 2):O27.

\footnotetext{
* Correspondence: I.staib@klinikum-esslingen.de

${ }^{1}$ Department of General and Visceral Surgery, Klinikum Esslingen, Esslingen, Germany

Full list of author information is available at the end of the article
} 